\title{
First Whole Transcriptome RNAseq on CHD8 Haploinsufficient Patient and Meta-Analyses Across Cellular Models Uncovers Likely Key Pathophysiological Target Genes
}

\author{
Heba Yasin ${ }^{1}$, Robert Stowe ${ }^{2}$, Chi Kin Wong ${ }^{3}$, Puthen Veettil Jithesh ${ }^{1}$, Farah R. Zahir ${ }^{3}$ \\ 1. Life Science, Hamad Bin Khalifa University, Doha, QAT 2. Psychiatry and Neurology, University of British Columbia, \\ Vancouver, CAN 3. Medical Genetics, University of British Columbia, Vancouver, CAN
}

Corresponding author: Farah R. Zahir, farahz@bcchr.ca

\begin{abstract}
In 2019, we confirmed that the haploinsufficiency of CHD8 does indeed cause the novel syndromic neurodevelopmental disease we first discovered a dozen years before. Here, we report the first whole transcriptome RNAseq gene expression profiling for a patient with this new syndrome, as a preliminary exploration of potential pathophysiological mechanisms. We compared our patient transcriptome profile with that of all publicly available RNAseq datasets from human cellular models including neuronal progenitor cells, neurons and organoids. We compared differential gene expression profiles overall and conducted phenotype-informed data filtration based on the characteristic syndrome presentation. We found that concordance among differential gene expression profiles was poor across all datasets. Nevertheless, remarkably, we show that the patient blood differential gene expression profile most resembled that of the neuronal cell model, a finding that encourages further transcriptome profiling using patient blood samples. In addition, our custom phenotype-informed analyses yielded important, differentially expressed syndrome pathophysiology target genes. Finally, we note that genes dysregulated due to CHD8 heterozygous deletion are linked to known neurological as well as oncological pathways.
\end{abstract}

Categories: Genetics, Pediatrics, Other

Keywords: chd8, intellectual disability, neurodevelopmental disorders, autism spectrum disorder, rnaseq

\section{Introduction}

In 2007, we first reported that the haploinsufficiency of CHD8 likely is syndromic for neurodevelopmental disease (ND) [1]. In the dozen years that followed, several others also published reports of CHD8 causation for neurodevelopmental conditions, including developmental delay (DD), intellectual disability (ID) and autism spectrum disorder (ASD) (see [2] for a review of these studies). Recently, we conducted a comprehensive review of all reported patients [2], and established that the haploinsufficiency of CHD8 is indeed causative for a novel neurodevelopmental syndrome, which we propose be called Zahir-Friedman syndrome (ZFS) [3]. The syndrome presentation includes DD and/or ID and/or ASD and macrocephaly,

Review began 10/14/2020 Review ended 11/18/2020 Published 11/19/2020

() Copyright 2020

Yasin et al. This is an open access article distributed under the terms of the Creative Commons Attribution License CC-BY 4.0., which permits unrestricted use, distribution, and reproduction in any medium, provided the original author and source are credited. speech delay, gastrointestinal (GI) problems, sleep problems, and skeletal and motor problems as characteristic, along with a distinguishing, if subtle, facial gestalt [2].

CHD8 is a chromatin remodeling gene with wide effects on gene regulation and transcription. Following the reports of $\mathrm{CHD} 8+$ /- as causative for ND [1], a bourgeoning interest in the gene led to the publication of key functional studies; important among them, transcriptional exploration in human cellular models - induced pluripotent stem cell derived-neuronal progenitor cells (NPC) [4-6], neurons [6] and cerebral organoids [7]. Yet, none of these studies explored the specific phenotypic outcomes presented by the syndrome in their analyses.

Here, we present the first whole transcriptome RNAseq analyses of a peripheral blood mononuclear cell (PBMC) sample for a ZFS patient. We chose patient Vancouver 4444, who has the smallest copy number variant (CNV) overlapping the critical region [2] and compared whole-genome expression profiles for him versus his normal age and sex-matched sibling in triplicate for this preliminary exploratory study. We have previously verified that this patient's CNV results in halved CHD8 gene expression [2]. In order to meaningfully assess the patient blood-derived transcriptome, we obtained complete whole transcriptome datasets from all published human induced pluripotent stem cells (iPSC)-derived CHD8+/- model studies available at the time and compared our patient's data to them [4-7]. We scrutinize all datasets using a custom analysis based on syndrome pathophysiology. By doing so, we are able to highlight key downstream targets of CHD8 that potentially play a role in the development of this syndrome.

\section{Materials And Methods}

Patient RNAseq 
Whole blood was drawn into ethylenediaminetetraacetic acid (EDTA) tubes from the patient and his normal brother (age and sex-matched control). PBMCs were isolated and total RNA was extracted using TRIzol and RNeasy kits (Qiagen; Hilden, Germany), as previously described [2]. Whole transcriptome RNAseq on the patient and his normal brother was performed in triplicate by the Biomedical Research Center at the University of British Columbia (BRC-UBC) sequencing platform as per their standard protocol. Samples were quality checked using the Agilent 2100 bioanalyzer (Agilent Technologies, Santa Clara, CA). Samples were then prepped following the standard protocol for the NEBnext Ultra II Standard mRNA kits (New England Biolabs, Ipswich, MA). Sequencing was performed on the Illumina NextSeq 500 (San Diego, California) with paired-end $42 \mathrm{bp} \mathrm{X} 42 \mathrm{bp}$ reads. Bioinformatic data analysis was generated by the BRC-UBC as per their standard analysis pipeline: de-multiplexed read sequences were aligned to the reference sequence using STAR (http://code.google.com/p/rna-star/). Cufflinks were used to estimate differential expression. RNAseq data were reposited at the European Nucleotide Archive and available at https://www.ebi.ac.uk/ega/datasets/EGAD00001006006.

\section{Selection of publicly available RNAseq data}

We selected five publicly available human cellular whole transcriptome datasets, comprising a total of 26 samples and 18 control datasets from five separate RNAseq datasets from four publications [4-7]. Appendix 1 tabulates laboratory methods and bioinformatics pipelines for the studies.

\section{Selection of differentially expressed genes (DEGs)}

DEGs were selected based on q-values generated by the Benjamini-Hochberg correction using a uniform threshold of $\mathrm{q}<0.05$ for each study.

\section{Obtaining phenotype seed genes (PSGs)}

The Phenolyzer (http://phenolyzer.wglab.org/, accessed January-May 2018) was used to generate a list of candidate genes termed a 'phenotype seed gene (PSG) list' for each of the 12 characteristic syndrome phenotypes. PSGs were generated using the search terms, as described in Appendix 2. PSGs obtained per phenotype are given in Appendix 3.

\section{Pathway analyses}

Pathway analyses were conducted for DEGs using Ingenuity Pathway Analysis (IPA®) from Ingenuity (http://www.ingenuity.com) as described in Appendix 2.

\section{Results}

\section{Patient RNAseq results}

Close to Half of the Expressed Genes Are Differentially Expressed in the Patient

Patient RNA sequencing detected 13,002 genes and 21,493 transcripts (Appendix 3 contains tables of expression levels for all detected genes). Differential gene expression analysis between patient and control revealed 5,388 significant DEGs ( $41 \%$ of all detected genes) at Benjamini-Hochberg $\mathrm{q}<0.05$, separating patient and control samples distinctively (Figure 1). 


\section{Cureus}
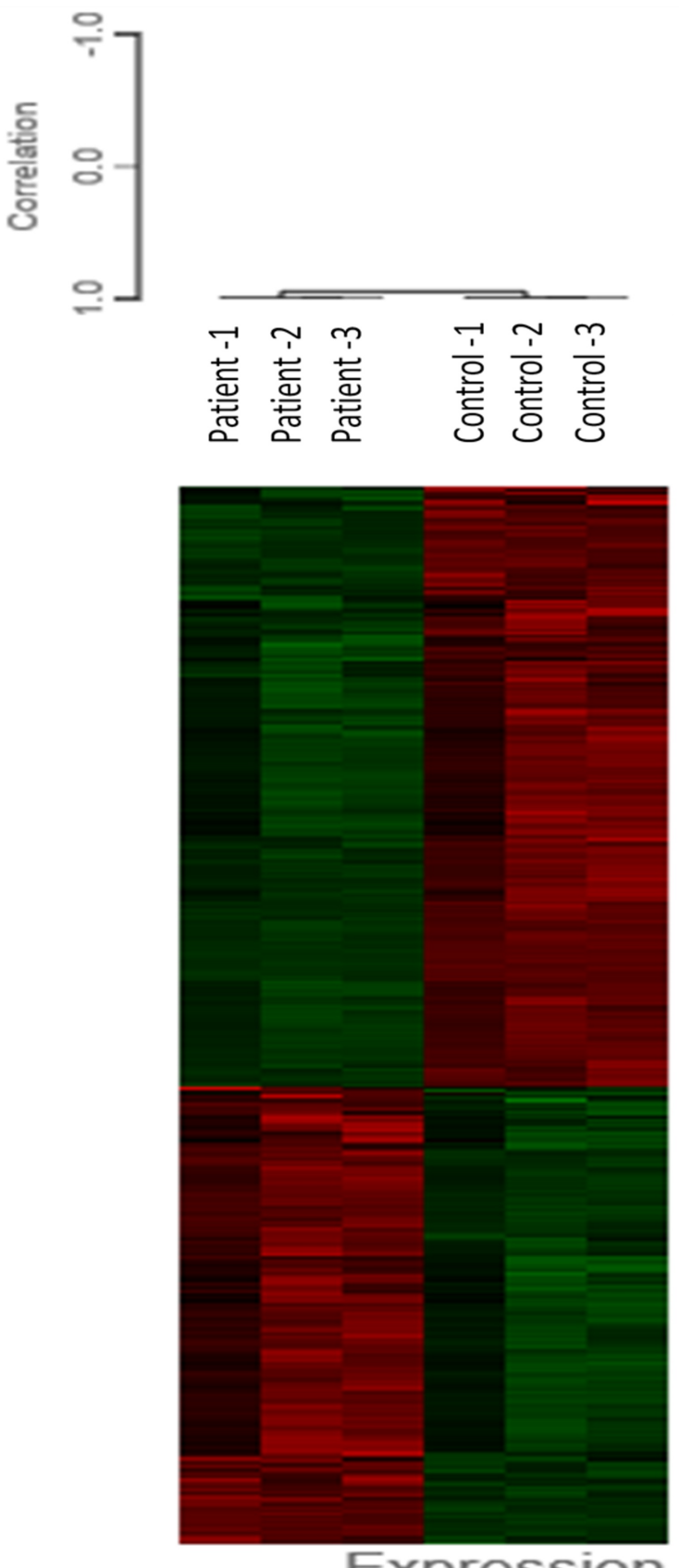

Expression

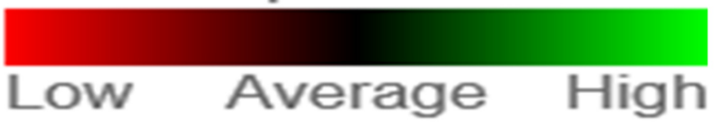

FIGURE 1: Whole transcriptome RNAseq heatmap for differential expression between patient and control samples

Slightly More Genes Were Upregulated (52\%) Than Downregulated (48\%)

Of the 5,388 DEGs in the patient, 386 had a fold change greater or equal to $\pm 1,64$ had a fold change greater or equal to \pm 2 , and 23 had a fold change \pm 3 (Figures $2 A-2 B$ and Table 1 ). Both the most upregulated and most downregulated DEGs in the patient are pseudogenes belonging to the ubiquitin peptidase family. Only three of the 23 genes in Table 1 appear as DEGs in any of the other datasets we analyzed (Appendix 3). 


\section{Cureus}

A

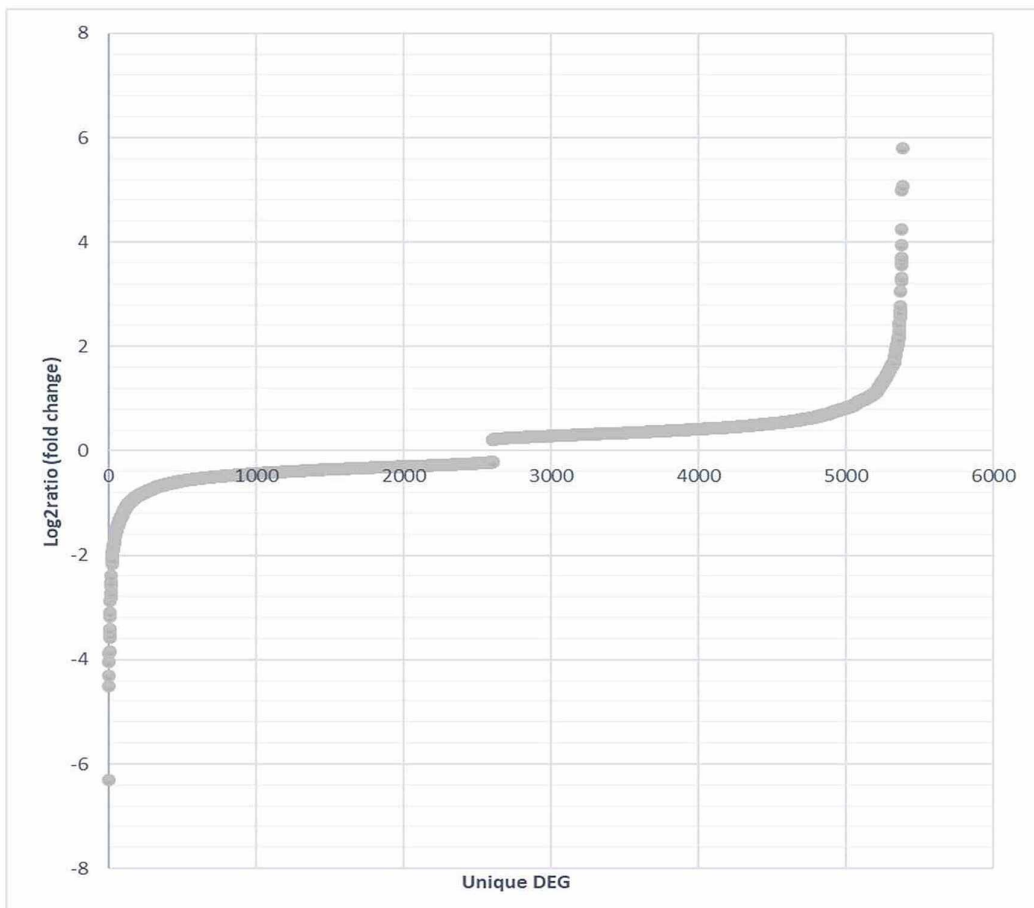

B

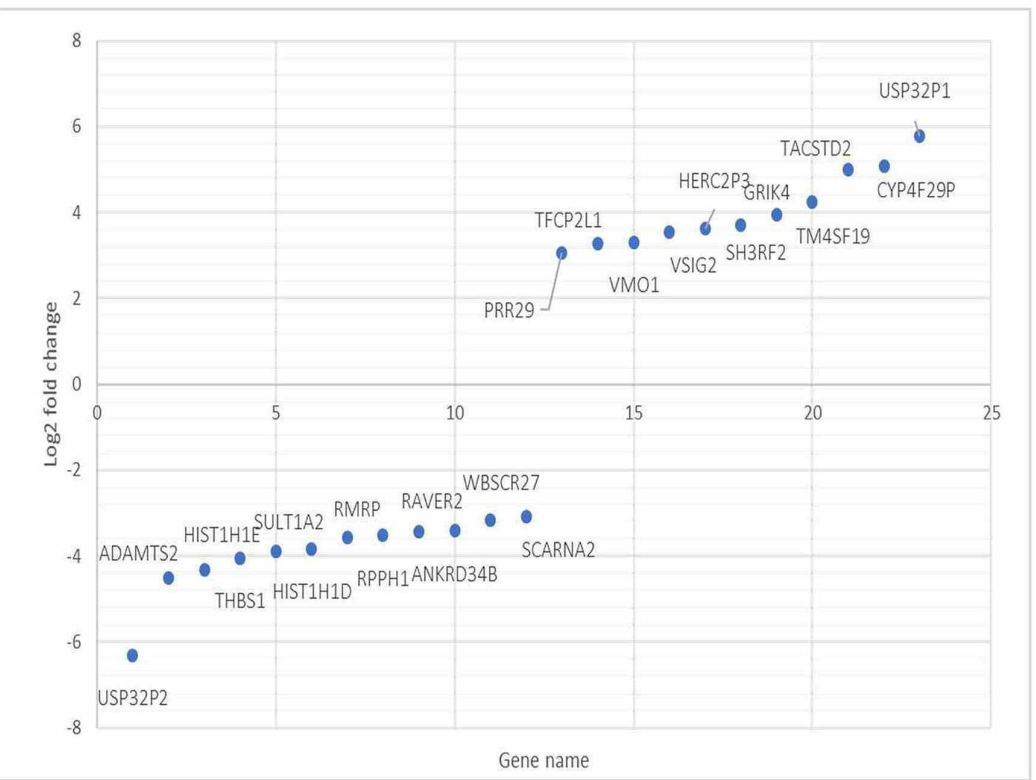

FIGURE 2: A - log2 fold change scatter plot showing differential gene expression between patient and control across all 5,388 detected transcripts. B -23 genes that showed a log 2 fold change \pm 3 between patient and control 


\section{Cureus}

\begin{tabular}{|c|c|c|c|c|c|}
\hline GENE & $\begin{array}{l}\text { LOG2 } \\
\text { (FOLD_CHANGE) }\end{array}$ & P_VALUE & Q_VALUE & $\begin{array}{l}\text { REGULATION IN } \\
\text { PATIENT }\end{array}$ & OTHER DATASETS GENE IS A DEG IN \\
\hline USP32P2 & -6.312 & 5.00E-05 & 0.000316967 & down & None \\
\hline ADAMTS2 & -4.49852 & 0.00025 & 0.00129193 & down & Wang_Neurons \\
\hline HIST1H1E & -4.31541 & 5.00E-05 & 0.000316967 & down & None \\
\hline THBS1 & -4.04863 & $5.00 \mathrm{E}-05$ & 0.000316967 & down & $\begin{array}{l}\text { Wilkinson, Wang_NPC and } \\
\text { Wang_Neurons }\end{array}$ \\
\hline SULT1A2 & -3.87789 & 0.00655 & 0.019417 & down & None \\
\hline HIST1H1D & -3.83365 & 0.00015 & 0.000841735 & down & Wilkinson \\
\hline RMRP & -3.57236 & 5.00E-05 & 0.000316967 & down & None \\
\hline RPPH1 & -3.5139 & $5.00 \mathrm{E}-05$ & 0.000316967 & down & None \\
\hline RAVER2 & -3.42626 & 0.0004 & 0.00193051 & down & None \\
\hline ANKRD34B & -3.41265 & 0.00035 & 0.00172049 & down & None \\
\hline WBSCR27 & -3.16821 & 0.0077 & 0.0223024 & down & None \\
\hline SCARNA2 & -3.09253 & 5.00E-05 & 0.000316967 & down & None \\
\hline PRR29 & 3.06369 & 0.00145 & 0.00555477 & up & None \\
\hline TFCP2L1 & 3.26474 & $5.00 \mathrm{E}-05$ & 0.000316967 & up & None \\
\hline VMO1 & 3.3094 & 5.00E-05 & 0.000316967 & up & None \\
\hline VSIG2 & 3.55333 & 0.0196 & 0.0477048 & up & None \\
\hline HERC2P3 & 3.63294 & 5.00E-05 & 0.000316967 & up & None \\
\hline SH3RF2 & 3.70726 & 0.00385 & 0.0124584 & up & None \\
\hline GRIK4 & 3.95099 & 0.0002 & 0.00107189 & up & None \\
\hline TM4SF19 & 4.23945 & 0.00115 & 0.00461064 & up & None \\
\hline TACSTD2 & 4.99907 & 0.0026 & 0.00902676 & up & None \\
\hline CYP4F29P & 5.07494 & 0.0001 & 0.000592077 & up & None \\
\hline USP32P1 & 5.78815 & $5.00 \mathrm{E}-05$ & 0.000316967 & up & None \\
\hline
\end{tabular}

TABLE 1: DEGs in patients that are expressed with a log2 fold change of either $<=-3$ or $<=3$ DEGs: differentially expressed genes

The Ubiquitination Proteasome Pathway (UPP) is the Most Differentially Expressed Pathway in Patients

IPA was used to explore differentially expressed canonical pathways and networks in the patient by inputting all patient DEGs. Of the 5,388 DEGs, all mapped in IPA, except for 11 uncharacterized, long, intergenic, non-protein-coding RNAs, which were unrecognized. Table 2 lists the top 10 differentially expressed pathways in the patient, with the UPP shown to be the most differentially expressed. M-TOR signaling is the next most perturbed. Among affected networks, the most affected is "Developmental Disorder, Hereditary Disorder, Neurological Disease” (Appendix 4). 


\section{Cureus}

\begin{tabular}{|l|l|}
\hline Ingenuity Canonical Pathways & -log (p-value) \\
\hline Protein Ubiquitination Pathway & 9.31 \\
mTOR Signaling & 8.58 \\
NF-KB Signaling & 7.94 \\
TNFR1 Signaling & 7.63 \\
Role of PKR in Interferon Induction and Antiviral Response & 7.4 \\
TNFR2 Signaling & 7.19 \\
TWEAK Signaling & 7.03 \\
Germ Cell-Sertoli Cell Junction Signaling & 6.99 \\
IL-8 Signaling & 6.48 \\
EIF2 Signaling & 6.39
\end{tabular}

TABLE 2: Top 10 differentially expressed pathways in the patient versus normal control

On Average, Over a Quarter of All PSGs Across Phenotypes Are DEGs in Patients

We then sought to investigate the involvement of DEGs in possible phenotype-developmental pathophysiology by investigating which DEGs are PSGs. An average of 27.3\% of genes from each phenotype seed gene list was found as a DEG in the patient (Table 3 and Figure 3), corresponding to an average of $3.2 \%$ of patient DEGs being included in each phenotype seed gene list. The highest number was found for the ID phenotype, having 327 patient DEGs that were also in this phenotype seed gene list (6\% of the total patient DEGs). In total, 724 of the patient's 5,388 DEGs were included as one or more PSGs. Appendix 3 contains tables that give a sample-based and phenotype-based summary of overlaps between DEGs and PSGs across all samples and phenotypes. 


\section{Cureus}

\begin{tabular}{|c|c|c|c|c|}
\hline Phenotype & $\begin{array}{l}\text { Patient DEGs that } \\
\text { are PSGs }\end{array}$ & $\begin{array}{l}\text { Total } \\
\text { PSG\# }\end{array}$ & $\begin{array}{l}\text { Percentage PSGs that are } \\
\text { patient DEGs }\end{array}$ & $\begin{array}{l}\text { Percentage of total patient DEGs (5388) } \\
\text { that are PSGs }\end{array}$ \\
\hline $\begin{array}{l}\text { Facial } \\
\text { dysmorphisms }\end{array}$ & 212 & 832 & $25.50 \%$ & $3.90 \%$ \\
\hline DD & 240 & 833 & $28.80 \%$ & $4.50 \%$ \\
\hline ID & 327 & 1236 & $26.50 \%$ & $6.10 \%$ \\
\hline ASD & 113 & 482 & $23.40 \%$ & $2.10 \%$ \\
\hline Speech delay & 124 & 494 & $25.10 \%$ & $2.30 \%$ \\
\hline Macrocephaly & 103 & 378 & $27.20 \%$ & $1.90 \%$ \\
\hline Pes planus & 62 & 181 & $34.30 \%$ & $1.20 \%$ \\
\hline Hypotonia & 258 & 937 & $27.50 \%$ & $4.80 \%$ \\
\hline Gl & 206 & 740 & $27.80 \%$ & $3.80 \%$ \\
\hline Sleep & 90 & 352 & $25.60 \%$ & $1.70 \%$ \\
\hline Anxiety & 101 & 372 & $27.20 \%$ & $1.90 \%$ \\
\hline Dental & 235 & 824 & $28.50 \%$ & $4.40 \%$ \\
\hline
\end{tabular}

\section{TABLE 3: Patient DEG overlap with PSG details}

DEG: differentially expressed gene; PSG: phenotype seed gene; DD: developmental delay; ID: intellectual disability; ASD: autism spectrum disorder; Gl: gastrointestinal

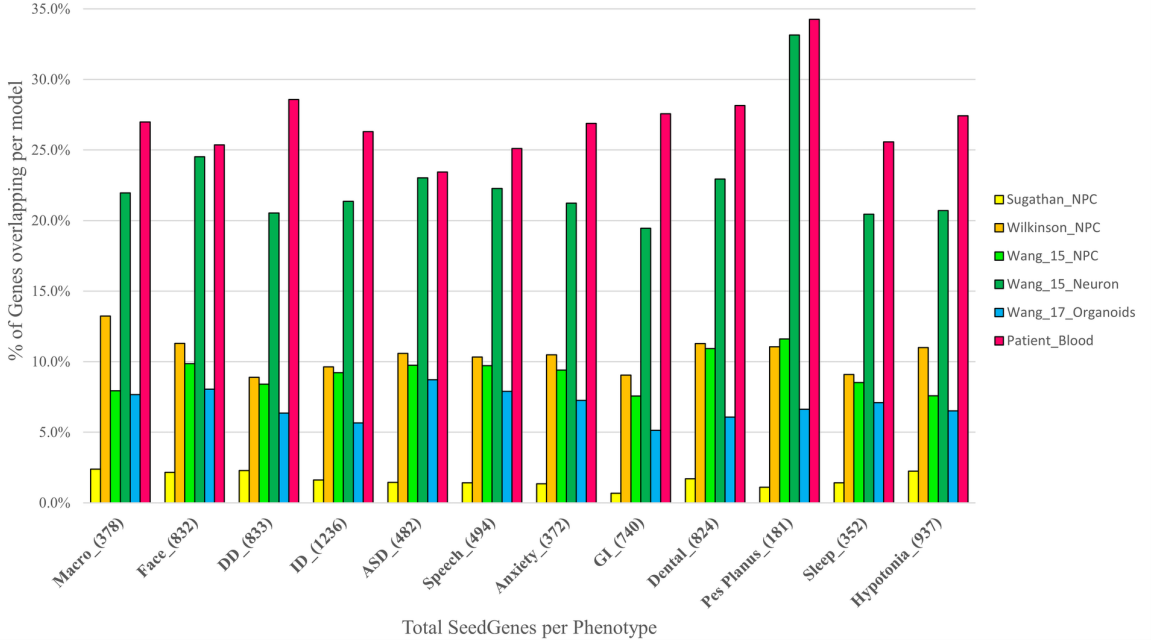

FIGURE 3: Bar plot showing the percentage of DEGs that appear as PSGs in each study, by phenotype

DEGs: differentially expressed genes; PSGs: phenotype seed genes

Patient DEGs That Are PSGs for Multiple Phenotypes Include Well-Recognized ND Causative Genes 


\section{Cureus}

We looked for patient DEGs that appear in over one PSG list as possibly important candidates that may influence a wide spectrum of phenotype pathophysiology. Sixteen DEGs in the patient were PSGs in at least nine of the 12 phenotypes (Table 4). Of these, CDKN1C and MECP2 are PSGs in 11 out of the 12 phenotypes. CDKN1C appearing as a PSG in all but speech defects and MECP2 appearing as a PSG in all but pes_planus (Appendix 3). WBSCR27 is the most deregulated with a three-fold reduction in expression while CDKN1C is the next deregulated, with a one-fold increase in expression.

\begin{tabular}{|c|c|c|c|}
\hline Patient DEGs & Total times found out of the 12 phenotypes & Log2 (fold change) & Regulation \\
\hline CDKN1C & 11 & 1.10845 & up \\
\hline MECP2 & 11 & 0.37637 & up \\
\hline NFIX & 10 & 0.413196 & up \\
\hline LIMK1 & 9 & 0.563392 & up \\
\hline FLNA & 9 & 0.535682 & up \\
\hline BCL7B & 9 & 0.419455 & up \\
\hline WBSCR16 & 9 & 0.30959 & up \\
\hline FLII & 9 & 0.23419 & up \\
\hline COG6 & 9 & -0.334932 & down \\
\hline NRAS & 9 & -0.34015 & down \\
\hline TCF4 & 9 & -0.351494 & down \\
\hline ATRX & 9 & -0.377614 & down \\
\hline KRAS & 9 & -0.427387 & down \\
\hline PTEN & 9 & -0.524188 & down \\
\hline DHFR & 9 & -0.565876 & down \\
\hline WBSCR27 & 9 & -3.16821 & down \\
\hline
\end{tabular}

TABLE 4: Patient DEGs that are frequent PSGs

DEGs: differentially expressed genes; PSGs: phenotype seed genes

\section{Comparison of the patient transcriptome with cellular model transcriptomes}

The DEG landscape across the patient sample and all models is discordant, with only FBXL19 and EML6 appearing as DEGs in all datasets: We compared the number and distribution of DEGs across all cellular samples and the patient's transcriptome. DEGs that met the expression threshold of $\mathrm{q}>0.5$ in all models and in the patient are given in Appendix 3. The most DEGs were found in the patient $(5,388)$ while only considering the cellular models, the most DEGs were identified by Wang et al. in neuron cells (3289). Sugathan et al. had the least (369) in NPCs. On average, 2,157 DEGs were found across all samples, with a median number of 1,530 DEGs (Table 5). 


\section{Cureus}

\section{Model}

Patient blood

Neurons_Wang 2015

NPC_Wilkinson

NPC_ Wang 2015

Organoids_Wang 2017

NPC_Sugathan

DEGs Average

DEGs Median

\section{Number of DEGs}

5388

3289

1812

1248

838

369

2157

1530

TABLE 5: Total DEG counts

DEG: differentially expressed gene

We sought to determine the overlap among the DEGs identified across all datasets. Concordance was poor among all transcriptomes, with only FBXL19 and EML6 as concordant DEGs across all datasets. Omitting Sugathan et al., we found CELF2, PODXL, RGMB, NFIA, and TCF4 were differentially expressed in the four cellular model datasets and in the patient transcriptome. There are no common DEGs to all the five cellular transcriptomes that are not found in the patient. RIMS3 was the only other gene common to four cellular models and the patient; it was not a DEG only in the Wang organoid sample. Of the eight genes above, we note that FBXL19 and EML6, as well as CELF2, PODXL, and RIMS3, are not seed genes in any of our PSG lists while TCF4, NFIA, and RGMB are PSGs (Table 6).

\begin{tabular}{|c|c|c|c|c|c|c|c|c|c|c|c|c|c|}
\hline & Macro & Face & DD & ID & ASD & Speech & Anxiety & GI & Dental & PP & Sleep & Hypotonia & Non-phenotype \\
\hline Patient & & TCF4 & TCF4 & TCF4 & $\begin{array}{l}\text { TCF4 } \\
\text { RGMB }\end{array}$ & TCF4 & NFIA & $\begin{array}{l}\text { TCF4 } \\
\text { NFIA }\end{array}$ & TCF4 & TCF4 & & $\begin{array}{l}\text { TCF4 } \\
\text { NFIA }\end{array}$ & $\begin{array}{l}\text { CELF2, EML6, FBXL19, PODXL, } \\
\text { RIMS3 }\end{array}$ \\
\hline $\begin{array}{l}\text { Wang } \\
\text { Organoid }\end{array}$ & & TCF4 & TCF4 & TCF4 & $\begin{array}{l}\text { TCF4 } \\
\text { RGMB }\end{array}$ & TCF4 & NFIA & $\begin{array}{l}\text { TCF4 } \\
\text { NFIA }\end{array}$ & TCF4 & TCF4 & & $\begin{array}{l}\text { TCF4 } \\
\text { NFIA }\end{array}$ & CELF2, EML6, FBXL19, PODXL \\
\hline Wang Neurons & & TCF4 & TCF4 & TCF4 & $\begin{array}{l}\text { TCF4 } \\
\text { RGMB }\end{array}$ & TCF4 & NFIA & $\begin{array}{l}\text { TCF4 } \\
\text { NFIA }\end{array}$ & ICF4 & CFF4 & & $\begin{array}{l}\text { TCF4 } \\
\text { NFIA }\end{array}$ & $\begin{array}{l}\text { CELF2, EML6, FBXL19, PODXL, } \\
\text { RIMS3 }\end{array}$ \\
\hline Wang NPC & & TCF4 & TCF4 & TCF4 & $\begin{array}{l}\text { TCF4 } \\
\text { RGMB }\end{array}$ & TCF4 & NFIA & $\begin{array}{l}\text { TCF4 } \\
\text { NFIA }\end{array}$ & TCF4 & TCF4 & & $\begin{array}{l}\text { TCF4 } \\
\text { NFIA }\end{array}$ & $\begin{array}{l}\text { CELF2, EML6, FBXL19, PODXL, } \\
\text { RIMS3 }\end{array}$ \\
\hline Wilkinson & & TCF4 & TCF4 & TCF4 & $\begin{array}{l}\text { TCF4 } \\
\text { RGMB }\end{array}$ & TCF4 & NFIA & $\begin{array}{l}\text { TCF4 } \\
\text { NFIA }\end{array}$ & TCF4 & CF4 & & $\begin{array}{l}\text { TCF4 } \\
\text { NFIA }\end{array}$ & $\begin{array}{l}\text { CELF2, EML6, FBXL19, PODXL, } \\
\text { RIMS3 }\end{array}$ \\
\hline Sugathan & & & & & & & & & & & & & EML6, FBXL19, RIMS3 \\
\hline
\end{tabular}

TABLE 6: DEGs that are PSGs and found in at least five of the six samples, arranged according to phenotype

DEGs: differentially expressed genes; PSGs: phenotype seed genes; DD: developmental delay; ID: intellectual disability; ASD: autism spectrum disorder; Gl: gastrointestinal

The rate of overlap between PSGs and DEGs differs among the datasets: We then conducted a focused subanalysis, looking only at DEGs that are also PSGs. As with the overall overlap of DEGs among the RNAseq dataset, we found similar discordance when looking at those DEGs that were also PSGs. The highest percentage of phenotype seed gene involvement was found in the patient's RNAseq data while the lowest was from the Sugathan et al. dataset. However, we note the Wang neuron transcriptome showed a distribution most resembling that of the patient, with an average of $22.3 \%$ for the 12 PSG lists found as Wang neuron DEGs (Figure 3). 
TCF4, NFIA, and RGMB are differentially expressed PSGs common across the patient transcriptome and four of the five cellular model transcriptomes: There was no PSG that appeared as DEGs in all six transcriptome datasets. However, three PSGs - TCF4, NFIA, and RGMB - appear as DEGs in the patient and in four cellular transcriptomes, i.e., in all except the Sugathan dataset (Table 6 and Appendix 5). TCF4 is a phenotype seed gene for DD, ID, ASD, speech delay, GI problems, dental, Pes planus, hypotonia, and facial dysmorphisms; NFIA is a phenotype seed gene for anxiety, GI problems, and hypotonia; and RGMB appears as a phenotype seed gene for ASD.

\section{Discussion}

\section{Patient transcriptome shows widespread gene deregulation and affects key pathways}

Almost $50 \%$ of detected genes were differentially expressed in the patient versus his normal brother, a finding in keeping with other studies in models [4-7]. Thus, our finding further corroborates the growing evidence that CHD8 has widespread downstream targeting [8].

The fact that approximately half of all detected genes are differentially expressed, and that several dozen genes show over a three-fold expression change prompted us to conduct pathway analyses as an informative data interpretation approach. IPA analysis revealed the UPP to be the most altered in expression. The UPP is an important pathway in sculpting the developing nervous system, maintaining brain plasticity in response to environmental stimuli, and has been implicated in the causation of ND [9]. The UPP is implicated in the development of both pediatric and adult brain tumors [10]. Importantly, the link between the UPP and ASD has also been gaining attention [11]. Our results draw further attention to this pathway as important in ND across diagnostic boundaries.

The second most deregulated pathway is the MTOR signaling pathway. Indeed, it and the UPP are elevated in comparison to the next eight most deregulated pathways that cluster together (Table 2). The mTOR pathway is an upstream regulator of WNT signaling, which has been reported by a number of model studies in CHD8+/- and Chd8+/- to be among the topmost perturbed [4-7].

\section{Meta-analyses across all samples show poor concordance}

When considering all our transcriptome profiles together, only two genes, EML6 and FBXL19, appeared as DEGs across all six sample transcriptomes, exemplifying overall poor concordance (Figure 4). Differences in the sample source material, laboratory, and bioinformatic methods are among possible reasons for the discordance as we discuss them under the study limitations. Nevertheless, several genes with important functions are found commonly differentially expressed across samples (Figure 4 and Table 6 ). We discuss them briefly later in this section.

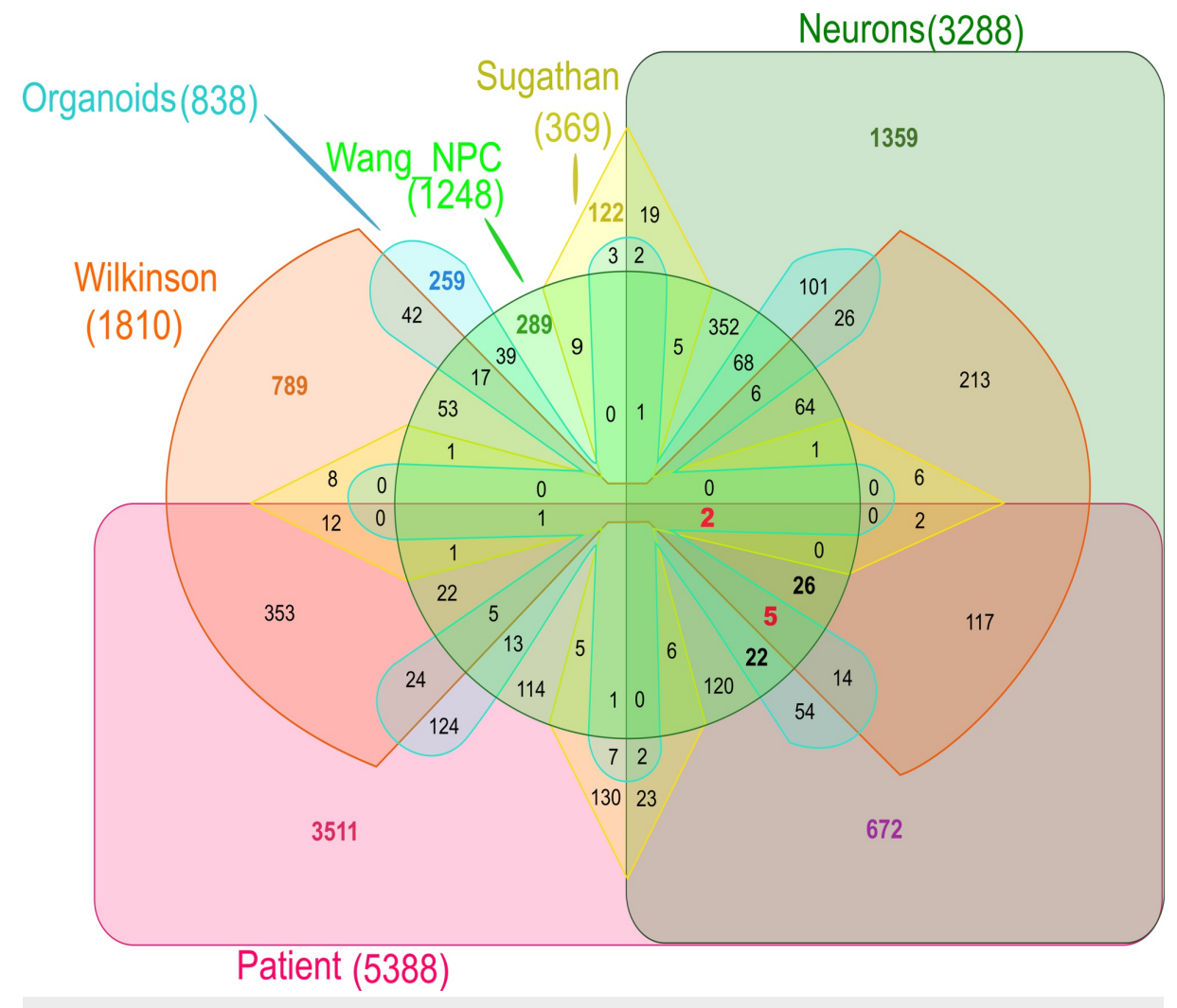




\section{Patient's blood transcriptome DEG profile most resembled that of the neuronal cell model}

Remarkably, the DEG profile of the neuronal model, and not that of NPCs, nor the organoid, most resembled that of our patient's transcriptome. The neuronal model produced the highest number of DEGs next to the patient transcriptome; 1,063 DEGs are common between the two datasets, of which, 672 were only found in only the patient and the neuronal transcriptome (Figure 4). Blood cells and neurons arise from different germ layers, the mesoderm, and ectoderm, respectively. Yet, consistent with our finding, a high degree of similarity is observed between the transcriptomes of blood and brain, with estimates of $35 \%$ to $80 \%$ of known transcripts to be present in both tissues and correlation in expression levels up to 0.64 observed [12]. In contrast, the lower concordance between blood and organoid transcriptome is surprising. While the complex differentiation signals undergone by neurons during organoid generation may play a part, we suggest this finding be explored further, as possible explanatory information is sparse.

\section{Syndrome-phenotype informed DEG filtration yields key candidate genes}

A key strength of our study is our informed phenotype-based data filtration approach. Having extensively cataloged the phenotypic spectrum of all reported ZFS patients [2], we utilized this knowledge as a means to identify possible key CHD8 targets. Twelve phenotypes are most commonly presented by ZFS patients as given in this paper's introduction (see Appendix 2 for the full list).

CHD8 is an epigenomic regulator [3] and haploinsufficiency is the causative mechanism for ZFS syndrome [2]. Genes already considered candidates for phenotypes in ZFS presentation, which we find to be significantly deregulated in either the patient or the cellular models, are therefore hypothesized to be good candidates for further study of ZFS manifestation.

Across the six transcriptomes and 12 phenotypes, the highest proportion of PSGs that were found as differentially expressed genes were found in the patient and then in the Wang neuronal sample. On average, a quarter of the PSG per phenotype was found in the DEGs of the patient transcriptome (Figure 3).

In the patient, the most upregulated and most downregulated PSGs found differentially expressed (Table 4) are both known to be causative for ND:

CDKN1C (cyclin-dependent kinase inhibitor 1C) encodes a tumor suppressor involved in growth control. It is located near the IC2 imprinting center. Reduction in the activity of CDKN1C is implicated in BeckwithWiedemann syndrome, an overgrowth syndrome. Conversely, maternal uni-parental isodisomy resulting in over-expression (as the maternal allele is hypomethylated) has been shown to cause IMAGe syndrome - a rare disease that includes intrauterine growth restriction, metaphyseal dysplasia, adrenal hypoplasia congenita, and genital anomalies [13]. In our patient, this gene had a one-fold increased expression as compared to the control. It is a PSG in all 12 phenotypes except for speech defects.

WBSCR27, also known as METTL27, is a gene located in a contiguous gene deletion region at chromosome 7q11.22-q11.23 considered to be causative for Williams-Beuren syndrome, a rare autosomal dominant ID syndrome that includes multi-system phenotypes and characteristic facies. WBSCR27 encodes a methyltransferase belonging to the ubiE/CoQ5 family. Curiously, none of the cellular models report WBSCR27 as differentially expressed (Appendix 3). WBSCR27 had a three-fold reduction of expression level in the patient. This gene is a PSG in all phenotypes except for macrocephaly, DD, and speech defects (Appendix 3).

\section{PSG involvement across all samples highlights potential lead pathophysiology target genes with a role in multiple phenotypes}

When we looked at PSG involvement across all samples (see Appendix 5 (A to L) for Venn diagrams of overlap per each phenotype), we were able to highlight a few genes that have high recurrence both among samples as well as among phenotypes (Table 6).

Only three PSGs - TCF4, NFIA, and RGMB - are differentially expressed in the patient and at least four other models. TCF4 is a PSG in nine of the 12 phenotypes, NFIA is a PSG in three, and RGMB is a PSG for only ASD (Table 6). We discuss them briefly below. 
TCF4 (transcription factor 4) encodes a member of the basic helix-loop-helix (bHLH) protein family that regulates transcription by binding promoters and enhancers. TCF4 appears to be a master driver for neurodevelopment, with demonstrated wide-range target-binding [14]. Common variants associated with schizophrenia and corneal endothelial dystrophy risk, and rare mutations causing the autosomal dominant Pitt-Hopkins syndrome [15] are reported. TCF4 has been well-studied as a regulator of neural differentiation and has known important functions in pathways associated with NDs, including ASD and ID [14]. Several features characteristic of Pitt-Hopkins syndrome are also found in our patient; DD, fine motor skills, gastrointestinal problems, and speech problems are common [2]. Emerging evidence shows TCF4 expression is a targetable prognostic indicator in cancer [16]. The TCF4 gene appears in all models excluding Sugathan et al. as a DEG and is a PSG in a remarkable nine of our 12 phenotypes (Table 6). These data and the known wide range of TCF4 involvement in cellular programming support the notion that a CHD8 haploinsufficiency-induced alteration in TCF4 expression is a key molecular signal in the pathophysiology of this condition.

RGMB (repulsive guidance molecule family member B) encodes a protein called DRAGON that is part of the repulsive guidance molecule (RGM) family of proteins. RGMs have been shown to play a role in the development and regulation of nervous and skeletal tissue and the gastrointestinal and immune system [17]. Oncogenic activity [18] and function during gut development for the mouse ortholog, as well as the modulatory activity for neurite outgrowth following spinal cord injury in rat [17], has been reported. We note the importance of DRAGON in fine control of brain homeostasis and its involvement in the gut as remarkable in ASD pathophysiology for which it is a PSG.

NFIA (Nuclear Factor I A) is one of the four-member nuclear factor 1 (NFI) transcription factors, that have important functions in the development of the brain among other organs. An autosomal dominant NFIArelated disorder with corpus callosum abnormalities and macrocephaly is reported, and it has been suggested to be the critical gene for the chromosome 1p32-p31 deletion syndrome [19].

In summary, our ZFS presentation informed data filtration approach conducted across these diverse samples has succeeded in drawing a spotlight onto what may be the key targets of CHD8 in disease pathophysiology for this syndrome. Further studies are warranted to determine the impact of the above genes on ZFS.

\section{Commonly differentially expressed genes that are not found as PSGs}

Recognizing that the definition of a PSG is entirely dependent on the corpus of accumulated data on gene function and pathology, we also carried out filtration for genes commonly differentially expressed across all samples that are not in any of the PSG candidates (Table 6). Two genes - EML6 and FBXL19 - were significantly differentially expressed in all six samples while two more - CELF2 and PODXL - were significantly differentially expressed in five samples excluding the low-yielding Sugathan transcriptome. Another gene, RIMS3 is notable for being differentially expressed in all samples except the organoid sample. We briefly discuss these genes below.

DEGs Common to All Six Samples

EML6 (echinoderm microtubule-associated protein-like 6) is a protein-coding gene of which not much is known. EML6 was found to localize on microtubules and showed high expression in E11 mouse embryos while declining in adults, which makes it a highly developmentally regulated gene that may be modifying the assembly dynamics of microtubules [20], precluding further comment.

FBXL19 (F-box and leucine-rich repeat protein 19) encodes a protein member of the E3 ubiquitin ligases. Several studies report that FBXL19 and its anti-sense mRNA, FBXL19-AS1, have important roles in various cancers [21]. In mice, it was recently shown that Fbxl19 is a CpG island-binding protein, enabling gene activation during cell lineage commitment, and is essential for development [22]. Thus, while FBXL19 was not selected as a PSG, its activity indicates an important, though as yet undefined, role in phenotype pathophysiology.

DEGs Common to All Samples Except the Sugathan Sample

CELF2 (CUGBP Elav-like family member 2) is a tumor suppressor that encodes CELF2, a protein that is one of the six-member CELF family of proteins that are recognized for their wide-ranging function in regulating pre-mRNA splicing and controlling the translation and stability of mRNAs [23]. While other family members have a more specific expression, CELF2 and CELF1 are broadly expressed [23]. There is strong evidence that CELF2 plays a role in myotonic dystrophy in particular and neurological disease in general [23]. CELF2 is a tumor suppressor with activity in cancers reported [24]. These data argue in favor of a possible wide-ranging influence for this gene on the pathophysiology of the syndrome. We particularly note this gene's involvement in motor function and consider this important with respect to the motor phenotypes seen in the syndrome, though it is not an identified PSG.

PODXL (podocalyxin-like) encodes a neural adhesion molecule and has been shown to cause autosomal 
recessive juvenile Parkinsonism [25]. A causative role for nephrosis and indicative roles in glomerular diseases [26], as well as a prognostic factor for some types of cancer [27], are known.

DEG Common to All Except the Organoid Sample

RIMS3 (regulating synaptic membrane exocytosis 3) encodes a protein thought to function in regulating synaptic membrane exocytosis. The fact that it does not appear as a DEG in the organoid sample but does so in all other samples likely indicates it is turned off later in differentiation.

In summary, this filtration has focused attention on candidate genes that require further study as important downstream targets of CHD8 in ZFS etiology. Additionally, looking at Table 6, we are able to obtain a preliminary insight into a potential gene expression cascade as we see DEGs that are common to samples spanning varied developmental time-points as well as specific phenotypes.

Common DEGs Are Also Cancer Susceptibility Genes

As noted above, many of these genes are cancer susceptibility genes. We previously noted the link to cancer for CHD8 haploinsufficiency, highlighting that the father of a patient with a paternally inherited CHD8 sequence mutation, who also had similar autistic features to the patient, developed cT2N1 rectum carcinoma [2]. Here, our findings of key DEGs that have wide-ranging involvement in both neurodevelopment and cancer further serve to underline the importance of cancer screening for patients with CHD8 haploinsufficiency.

\section{Study strengths and weaknesses}

While we have highlighted several interesting genes that may play a significant role in ZFS causation in this study, we caution that this is a preliminary investigation and more work is required to make stronger conclusions. We are limited by the number of patient samples used. We were uncertain whether using patient blood samples would allow us to meaningfully investigate the gene expression profile for a neurodevelopmental disorder. Therefore, taking this first patient's blood transcriptome, we compared it to five different human cellular models and were able to show that the patient profile most resembles a neuronal profile, leading the way for more patient blood transcriptomes to be profiled.

We note the variety of sample material used may confound results. The six transcriptome datasets include our patient's venous blood-derived PBMC sample, three NPC samples from different groups [4-6], a neuronal cell sample, and an organoid sample from the same group [6-7] (Appendix 1). Yet, among the three datasets that are all from NPCs, the data substantially differ (Figure 4), indicating a sample type bias may not play as much of a role as thought. Further having this variety in the developmental stage allowed us to unearth candidate genes important across developmental stages (Table 6).

We acknowledge there is a wide variety of experimental and bioinformatic methods used across all studies (Appendix 1). Remarkable differences include the advanced donor age in the Sugathan study (cells were derived from a 63-year-old human control subject). Donor age has been shown many times to impact reprogramming efficiency in mice [28] and may explain the markedly different results we obtained from the Sugathan sample profile. Also noteworthy is the fact that the Wang et al. datasets did not include CHD8 as significantly differentially expressed. They could not give an exact reason for this (personal communication). However, the CHD8 expression level reported by Wang is most close to a fold change of 0.5 in their neuronal model versus their NPC and their organoid models [7]. This explains why CHD8 does not appear as a common overlapping gene among all datasets for phenotypes such as ID, ASD, and speech delay, for which it is a well-documented known gene, as would be expected.

We were concerned that differences in bioinformatics data processing among the studies could confound results. However, Wade et al., who analyzed a larger series of publicly available datasets, including the five we used, and who subjected the raw sequence data to a uniform in-house bioinformatic pipeline, still report discordancy [8].

Taking these potential causes for variation into consideration, we reasoned that since uniform bioinformatic processing does not alleviate discordance and since we cannot cancel out the sample and experiment specific-bias inherent to each study, we would accept such study-dependent inherent bias and instead apply a uniform differential expression threshold of $q>0.5$ to define DEGs to be used in our meta-analysis, so that our acceptance threshold for DEGs would alleviate between study bias as much as possible.

Finally, we surmised that conducting the phenotype-informed focused analysis is a means to derive meaningful concordant data, as demonstrated by our findings. However, as with all exploratory analyses, we cannot rule out there could be other important target genes missed by our approach.

\section{Conclusions}




\section{Cureus}

Here we report the first whole transcriptome RNAseq analyses for a patient with ZFS. Comparing our patient transcriptome to five, different, human cellular engineered model transcriptomes, using both a noninformed and patient phenotype-informed analysis, we are able to unearth key biological pathways and downstream target genes that include those with known phenotypic involvement, as well as novel targets. We show that CHD8 haploinsufficiency in the patient causes widespread transcriptome expression change and affects a number of molecular pathways, as has been reported in both cellular and animal models. By examining the comparison between our patient transcriptome and cellular models that included neuronal progenitors, neurons, and brain-organoids, we strikingly discover that the patient blood transcriptome most resembles a neuronal cellular model. This finding is encouraging for further transcriptome profiling of patient blood samples as a means to profile this ND condition.

In summary, in this preliminary observational analysis, we are able to show patient blood transcriptomes are a viable option for further gene expression profiling of CHD8+/- impact on ND, and highlight important genes with both known and novel involvement with ZFS phenotypes that may potentially be key players in syndrome pathophysiology.

\section{Appendices}

\section{Appendix 1}

A table giving an overview of laboratory methods and bioinformatics pipelines used for each transcriptome dataset: 


\section{Cureus}

\begin{tabular}{|c|c|c|c|c|c|}
\hline Dataset & Patient & Sugathan NPCs & Wilkinson NPCs & $\begin{array}{l}\text { Wang NPCs and } \\
\text { Neurons }\end{array}$ & Wang Organoids \\
\hline $\begin{array}{l}\text { GEO } \\
\text { accession } \\
\text { no. }\end{array}$ & & GSE61492 & & GSE71594 & GSE8541/ \\
\hline Cell type & PBMCs & $\begin{array}{l}\text { Induced pluripotent } \\
\text { stem cells (iPSCs) } \\
\text { derived neural } \\
\text { progenitor cells (NPCs) } \\
\text { from human control } \\
\text { (GM8330) }\end{array}$ & $\begin{array}{l}\text { Commercial (SK-N-SH) human } \\
\text { NPCs from neuroblastoma cells }\end{array}$ & $\begin{array}{l}\text { iPSCs and neurons } \\
\text { developed from } \\
\text { human control } \\
\text { fibroblasts obtained } \\
\text { from a skin biopsy. }\end{array}$ & $\begin{array}{l}\text { Differentiated cerebral } \\
\text { organoids derived } \\
\text { from developed iPSC- } \\
\text { derived NPCs from } \\
\text { Wang et al., } 2015 .\end{array}$ \\
\hline Samples & $\begin{array}{l}3 \\
\text { samples } \\
3 \\
\text { controls }\end{array}$ & 14 samples 8 controls & 4 samples 4 controls & $\begin{array}{l}\text { NPCs: } 2 \text { samples, } 2 \\
\text { controls. Neurons: } 2 \\
\text { samples, } 2 \text { controls. }\end{array}$ & 4 samples 2 controls \\
\hline $\begin{array}{l}\text { Gene } \\
\text { knockdown } \\
\text { method }\end{array}$ & Native & $\begin{array}{l}\text { Transduction by HIV- } \\
\text { based lentiviral vectors } \\
\text { carrying shRNAs } \\
\text { targeting the CHD8 } \\
\text { gene. }\end{array}$ & $\begin{array}{l}\text { Transfection by siRNA silencer } \\
\text { selective negative control and } \\
\text { siRNA targeting the CHD8 gene }\end{array}$ & \multicolumn{2}{|c|}{$\begin{array}{l}\text { Transduction with CRISPR/Cas9 vectors } \\
\text { containing two separate CRISPR sgRNA } \\
\text { sequences to target the } \mathrm{N} \text {-terminal of CHD8 } \\
\text { protein to generate truncated mutations }\end{array}$} \\
\hline NGS & $\begin{array}{l}\text { Total } \\
\text { RNAseq }\end{array}$ & $\begin{array}{l}\text { Strand-specific dUTP } \\
\text { RNAseq. } 40.6 \text { million } \\
\text { reads per line at a final } \\
\text { depth } \sim 45 \text { million for } \\
\text { total reads. }\end{array}$ & $\begin{array}{l}\text { RNAseq focusing on long } \\
\text { noncoding RNAs. Average of } 45 \\
\text { million per knockdown replicate } \\
\text { and an average of } 40 \text { million } \\
\text { reads per control replicate. }\end{array}$ & Total RNAseq & Total RNAseq \\
\hline $\begin{array}{l}\text { Number of } \\
\text { DEGs }\end{array}$ & 5388 & 369 & 1812 & $\begin{array}{l}\text { NPCs: } 1248 \text {, } \\
\text { Neurons: } 3289\end{array}$ & 838 \\
\hline $\begin{array}{l}\text { Mapping/ } \\
\text { alignment: }\end{array}$ & & $\begin{array}{l}\text { Gsnap (GrCH37) } \\
\text { ensemble (71) }\end{array}$ & $\begin{array}{l}\text { transcriptome :UCSC } \\
\text { Hg19,Ensemble GrCH37 } \\
\text { (75),Tophat2 }\end{array}$ & $\begin{array}{l}\text { Tophat (hg19) } \\
\text { Gencode database, } \\
\text { Cufflink used to get } \\
\text { FPKM }\end{array}$ & $\begin{array}{l}\text { Kallisto using } \\
\text { GENCODE. TPM }>1 \text { in } \\
\text { WT samples }\end{array}$ \\
\hline DE analysis & & $\begin{array}{l}\text { DESeq (2 factor model) } \\
1.12 .1\end{array}$ & $\begin{array}{l}\text { Cuffdiff }+ \text { GTF file of the } \\
\text { transcriptome using default } \\
\text { settings }\end{array}$ & DESeq2 & DESeq2 \\
\hline $\begin{array}{l}\text { Functional } \\
\text { analysis }\end{array}$ & & DAVID, ToppGene & $\begin{array}{l}\text { DAVID, Disease association } \\
\text { protein-protein link evaluator }\end{array}$ & $\begin{array}{l}\text { DAVID, IPA, } \\
\text { ToppGene }\end{array}$ & DAVID, IPA \\
\hline
\end{tabular}

TABLE 7: An overview of laboratory methods and bioinformatics pipelines used by each dataset presented in this paper

\section{Appendix 2}

Notes describing (A) Phenotype seed gene list generation parameters and (B) IPA pathway analysis settings used in this study.

(A) Phenotype Seed Gene List Generation

The following search terms were entered into Phenolyzer (http://phenolyzer.wglab.org/) in order to obtain seed genes for each phenotype.

Phenotype 1: Facial dysmorphisms. The following unique terms were searched, and results amalgamated as PSGs for 'facial dysmorphisms': "auricular malformation", "cupid bow upper lip", "cupid bow shaped upper lip", "high palate”, "hypertelorism”, "long philtrum”, "prominent supraorbital ridges”, "down slanting palpebral fissure”, “down slanted palpebral fissures”, “downward slanting palpebral fissures”, "downward slanted palpebral fissures”. 
Phenotype 2: Behavioral presentations: “anxiety”.

Phenotype 3: DD: “developmental delay”.

Phenotype 4: ID: “intellectual disabilities”.

Phenotype 5: ASD: “autism”, “autistic”, “autism spectrum disorders”.

Phenotype 6: Macrocephaly: "Macrocephaly".

Phenotype 7: Speech complications: "speech delay”, "language development disorder". Phenotype 8: Skeletal complications: "pes planus".

Phenotype 9: Neurological complications: “hypotonia”.

Phenotype 10: GI complications: "diarrhea”, “constipation".

Phenotype 11: Sleep complications: "sleep disturbance”, “sleep disorder".

Phenotype 12: Dental complications: "dental anomalies"

(B) IPA Pathway Analyses:

Analysis creation date: 2018-04-26 and build version: 470319M.

Analysis settings:

. $\quad$ Reference set: Ingenuity Knowledge Base (Genes Only)

- Relationship to include: Direct and Indirect

- Includes Endogenous Chemicals

- Filter Summary:

Consider only relationships where (confidence $=$ High (predicted) OR Experimentally Observed) AND (data sources = An Open Access Database of Genome-wide Association Results OR BIND OR BioGRID OR ClinicalTrials.gov OR ClinVar OR Cognia OR DIP OR DrugBank OR Gene Ontology (GO) OR GVK Biosciences OR Hazardous Substances Data Bank (HSDB) OR HumanCyc OR Ingenuity Expert Findings OR Ingenuity ExpertAssist Findings OR IntAct OR Interactome studies OR MIPS OR miRBase OR miRecords OR Mouse Genome Database (MGD) OR Obesity Gene Map Database OR Online Mendelian Inheritance in Man (OMIM) OR TarBase OR TargetScan Human)

\section{Appendix 3}

Below is a list of tables and datasets that are too large to be appended. They are available upon request.

(A) Table of all phenotype seed genes organized by phenotype - 18-page PDF document.

(B) Table of all genes found expressed by RNAseq in the patient with log2fold change values and measures of statistical significance - 573-page PDF document.

(C) Table of all differentially expressed genes in the six transcriptome samples organized by sample - 69page PDF document.

(D) Multi-sheet Excel workbook containing a summary of overlaps between DEGs and PSGs organized by sample.

(E) Multi-sheet Excel workbook containing a summary of overlaps between DEGs and PSGs organized by phenotype.

\section{Appendix 4}

Pathway diagram for the most important network "Developmental Disorder, Hereditary Disorder, Neurological Disease” from the patient's RNAseq data 


\section{Cureus}

Network 1 : Patient_all_DEGs_all_cells - 2018-04-26 01:47 PM : Patient_all_DEGs_qValue_0.05 : Patient_all_DEGs_all_cells - 2018-04-26 01:47 F

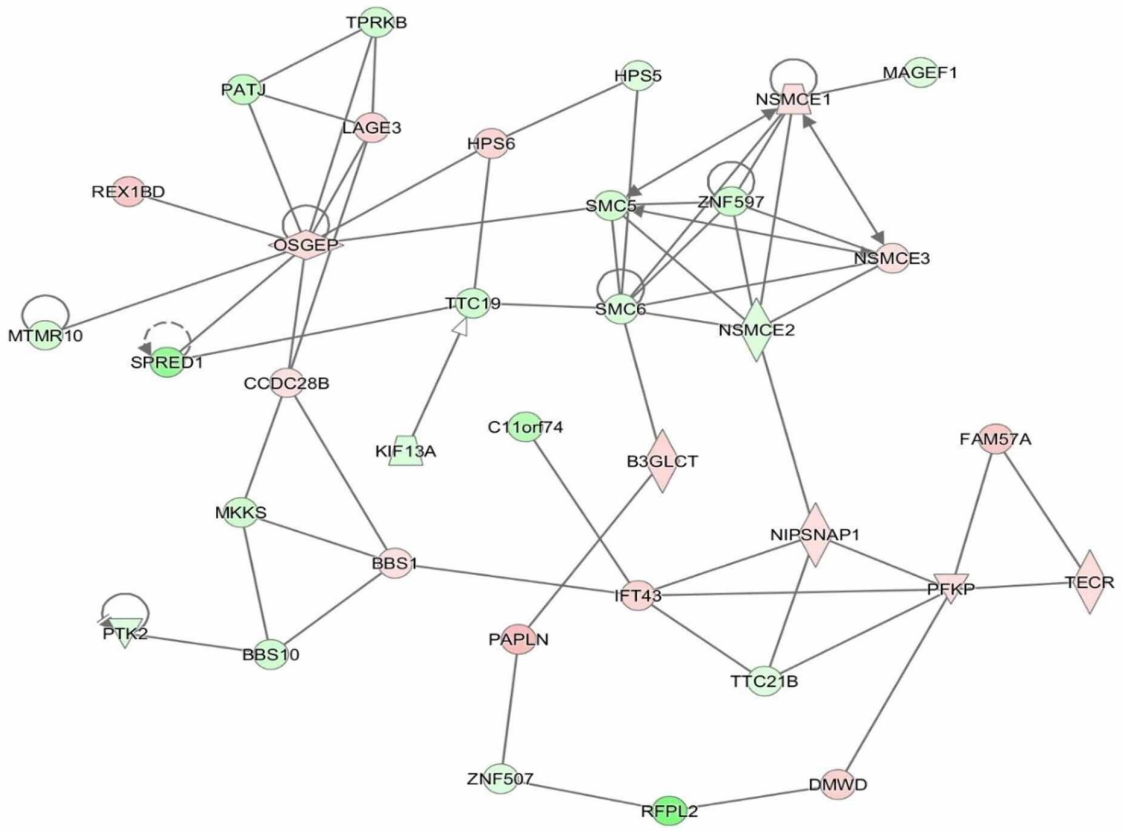

FIGURE 5: Connections and regulation of molecules found in the most important network "Developmental Disorder, Hereditary Disorder, Neurological Disease" from the patient's RNAseq data

\section{Appendix 5}

This Appendix contains two images (to maintain readability), which contain 12 panels, each depicting a sixway Venn diagram for intersections of all DEGs among samples by phenotype. Part A - panels A to F and Part B - panels G to L.

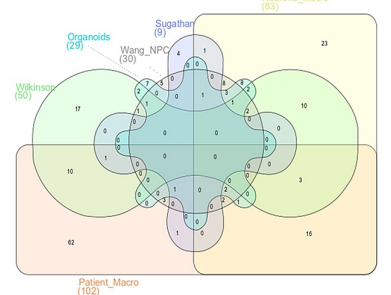

A- Macrocephaly

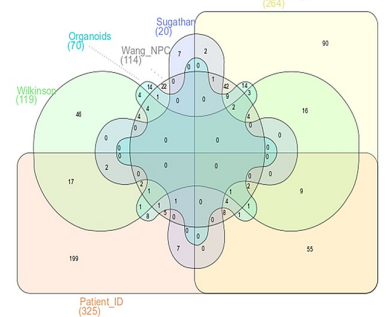

D- Intellectual Disability

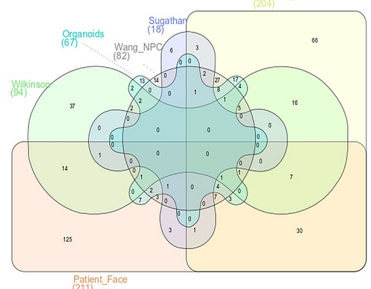

B - Facial Dyspmorphism

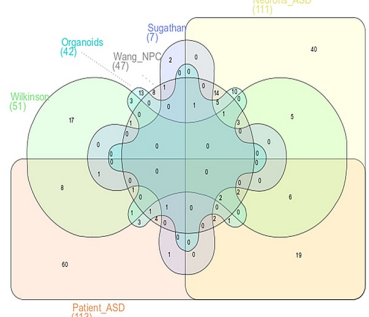

E - Autism Spectrum Disorder

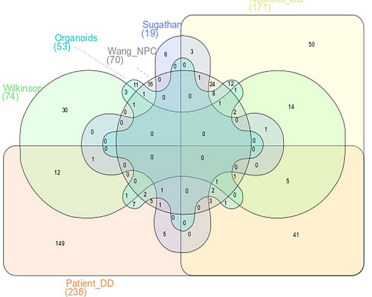

C-Developmental Disability

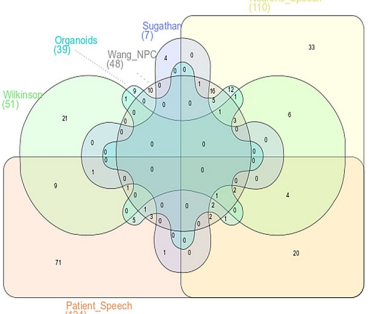

F-Speech Delay

FIGURE 6: Part A - six-way Venn intersections of DEGs across samples according to each phenotype

DEGs: differentially expressed genes 


\section{Cureus}

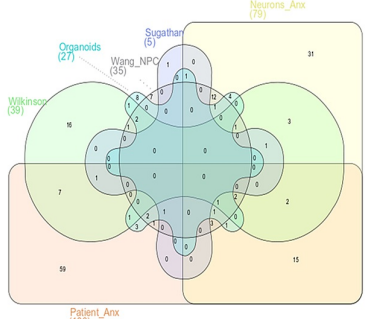

G - Anxiety

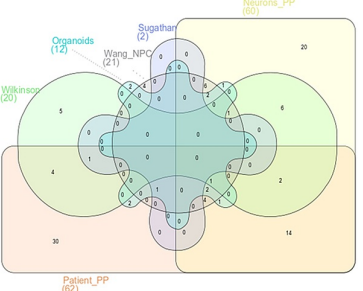

J - Pes Planus

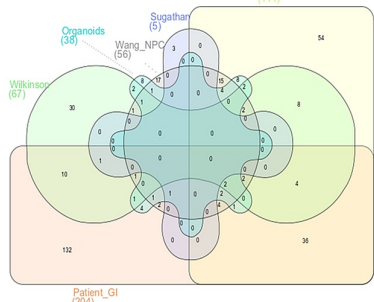

$\mathrm{H}$ - Gastrointestinal complications

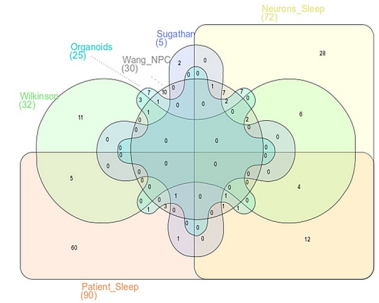

$\mathrm{K}$ - Sleep problems

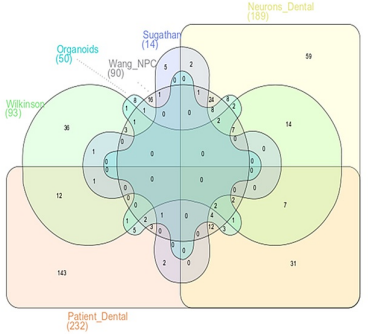

I - Dental anomalies

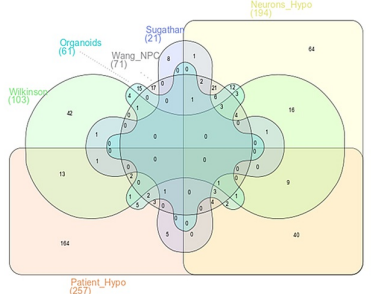

L - Hypotonia

FIGURE 7: Part B - six-way Venn intersections of DEGs across samples according to each phenotype

DEGs: differentially expressed genes

\section{Additional Information}

\section{Disclosures}

Human subjects: Consent was obtained by all participants in this study. BC Children's and Women's Hospital Research Ethics Board issued approval H09-01228. The patient was enrolled in the study of rare diseases project at the BC Children's and Women's Hospital. This study is approved by the BC Children's and Women's Hospital Research Ethics Boards (Institutional Review Board \# H09-01228). All participants provided informed consent. Animal subjects: All authors have confirmed that this study did not involve animal subjects or tissue. Conflicts of interest: In compliance with the ICMJE uniform disclosure form, all authors declare the following: Payment/services info: All authors have declared that no financial support was received from any organization for the submitted work. Financial relationships: All authors have declared that they have no financial relationships at present or within the previous three years with any organizations that might have an interest in the submitted work. Other relationships: All authors have declared that there are no other relationships or activities that could appear to have influenced the submitted work.

\section{Acknowledgements}

We thank our patient and his family for their participation in this study and their support; Dr. William Gibson and the staff at his laboratory for assistance rendered with sample collection; Dr. Imed Gallouzi and his laboratory for assistance with RNA sequencing; and Mr. Hakeem Almabrazi and Dr. Tanwir Habib for their assistance with data analysis.

\section{References}

1. Zahir F, Firth H V, Baross A, et al.: Novel deletions of $14 \mathrm{q} 11.2$ associated with developmental delay, cognitive impairment and similar minor anomalies in three children. J Med Genet. 2007, 44:556-561. 10.1136/jmg.2007.050823

2. Yasin H, Gibson WT, Langlois S, et al.: A distinct neurodevelopmental syndrome with intellectual disability, autism spectrum disorder, characteristic facies, and macrocephaly is caused by defects in CHD8. J Hum Genet. 2019, 64:271-280. 10.1038/s10038-019-0561-0

3. Yasin H, Zahir FR: Chromodomain helicase DNA-binding proteins and neurodevelopmental disorders . J Transl Genet Genom. 2020, 4:[Epub ahead of print]. 10.20517/jtgg.2020.30

4. Sugathan A, Biagioli M, Golzio C, et al.: CHD8 regulates neurodevelopmental pathways associated with autism spectrum disorder in neural progenitors. Proc Natl Acad Sci. 2014, 111:4468-4477. 10.1073/pnas.1405266111

5. Wilkinson B, Grepo N, Thompson BL, et al.: The autism-associated gene chromodomain helicase DNAbinding protein 8 (CHD8) regulates noncoding RNAs and autism-related genes. Transl Psychiatry. 2015, 5:e568. 10.1038/tp.2015.62 
6. Wang P, Lin M, Pedrosa E, et al.: CRISPR/Cas9-mediated heterozygous knockout of the autism gene CHD8 and characterization of its transcriptional networks in neurodevelopment. Mol Autism. 2015, 6:55. 10.1186/s13229-015-0048-6

7. Wang P, Mokhtari R, Pedrosa E, et al.: CRISPR/Cas9-mediated heterozygous knockout of the autism gene CHD8 and characterization of its transcriptional networks in cerebral organoids derived from iPS cells. Mol Autism. 2017, 8:1-17. 10.1186/s13229-017-0124-1

8. Wade AA, Lim K, Catta-Preta R, Nord AS: Common CHD8 genomic targets contrast with model-specific transcriptional impacts of CHD8 haploinsufficiency. Front Mol Neurosci. 2019, 11:481. 10.3389/fnmol.2018.00481

9. Zahir FR, Mwenifumbo JC, Chun H-JE, et al.: Comprehensive whole genome sequence analyses yields novel genetic and structural insights for intellectual disability. BMC Genomics. 2017, 18:403. 10.1186/s12864017-3671-0

10. Zaky W, Manton C, Miller CP, Khatua S, Gopalakrishnan V, Chandra J: The ubiquitin-proteasome pathway in adult and pediatric brain tumors: biological insights and therapeutic opportunities. Cancer Metastasis Rev. 2017, 36:617-633. 10.1007/s10555-017-9700-2

11. Vatsa N, Jana NR: UBE3A and its link with autism. Front Mol Neurosci. 2018, 4:448. 10.3389/fnmol.2018.00448

12. Tylee DS, Kawaguchi DM, Glatt SJ: On the outside, looking in: a review and evaluation of the comparability of blood and brain “-omes”. Am J Med Genet B Neuropsychiatr Genet. 2013, 162:595-603. 10.1002/ajmg.b.32150

13. Romanelli V, Belinchon A, Benito-Sanz S, et al.: CDKN1C (p57(Kip2)) analysis in Beckwith-Wiedemann syndrome (BWS) patients: Genotype-phenotype correlations, novel mutations, and polymorphisms. Am J Med Genet A. 2010, 152:1390-1397. 10.1002/ajmg.a.33453

14. Forrest MP, Hill MJ, Kavanagh DH, Tansey KE, Waite AJ, Blake DJ: The psychiatric risk gene transcription factor 4 (tcf4) regulates neurodevelopmental pathways associated with schizophrenia, autism, and intellectual disability. Schizophr Bull. 2018, 44:1100-1110. 10.1093/schbul/sbx164

15. Goodspeed K, Newsom C, Morris MA, Powell C, Evans P, Golla S: Pitt-Hopkins syndrome: a review of current literature, clinical approach, and 23-patient case series. J Child Neurol. 2018, 33:233-244. 10.1177/0883073817750490

16. Jain N, Hartert K, Tadros S, et al.: Targetable genetic alterations of TCF4 (E2-2) drive immunoglobulin expression in diffuse large B cell lymphoma. Sci Transl Med. 2019, 11:eaav5599. 10.1126/scitranslmed.aav5599

17. Siebold C, Yamashita T, Monnier PP, Mueller BK, Pasterkamp RJ: RGMs: structural insights, molecular regulation, and downstream signaling. Trends Cell Biol. 2017, 27:365-378. 10.1016/j.tcb.2016.11.009

18. Shi Y, Chen G-B, Huang X-X, et al.: Dragon (repulsive guidance molecule b, RGMb) is a novel gene that promotes colorectal cancer growth. Oncotarget. 2015, 6:20540-20554. 10.18632/oncotarget.4110

19. Rivera-Pedroza CI, Barraza-García J, Paumard-Hernández B, Nevado J, Orbea-Gallardo C, Sánchez del Pozo J, Heath KE: Chromosome 1p31.1p31.3 deletion in a patient with craniosynostosis, central nervous system and renal malformation: case report and review of the literature. Mol Syndromol. 2017, 8:30-35. $10.1159 / 000452609$

20. Houtman SH, Rutteman M, De Zeeuw CI, French PI: Echinoderm microtubule-associated protein like protein 4, a member of the echinoderm microtubule-associated protein family, stabilizes microtubules. Neuroscience. 2007, 144:1373-1382. 10.1016/j.neuroscience.2006.11.015

21. Shen B, Yuan Y, Zhang Y, Yu S, Peng W, Huang X, Feng J: Long non-coding RNA FBXL19-AS1 plays oncogenic role in colorectal cancer by sponging miR-203. Biochem Biophys Res Commun. 2017, 488:67-73. 10.1016/j.bbrc.2017.05.008

22. FBXL19 recruits CDK-mediator to $\mathrm{CpG}$ islands of developmental genes priming them for activation during lineage commitment. (2018). https://elifesciences.org/articles/37084.

23. New J, Subramaniam D, Ramalingam S, et al.: Pleotropic role of RNA binding protein CELF2 in autophagy induction. Mol Carcinog. 2019, 58:1400-1409. 10.1002/mc.23023

24. Wang J, Liu L, Sun Y, et al.: miR-615-3p promotes proliferation and migration and inhibits apoptosis through its potential target CELF2 in gastric cancer. Biomed Pharmacother. 2018, 101:406-413. 10.1016/j.biopha.2018.02.104

25. Sudhaman S, Prasad K, Behari M, Muthane UB, Juyal RC, Thelma BK: Discovery of a frameshift mutation in podocalyxin-like (PODXL) gene, coding for a neural adhesion molecule, as causal for autosomal-recessive juvenile Parkinsonism. J Med Genet. 2016, 53:450-456. 10.1136/jmedgenet-2015-103459

26. Akankwasa G, Jianhua L, Guixue C, Changjuan A, Xiaosong Q: Urine markers of podocyte dysfunction: a review of podocalyxin and nephrin in selected glomerular diseases. Biomark Med. 2018, 12:927-935. 10.2217/bmm-2018-0152

27. Zhi Q, Chen H, Liu F, et al.: Podocalyxin-like protein promotes gastric cancer progression through interacting with RUN and FYVE domain containing 1 protein. Cancer Sci. 2019, 110:118-134. 10.1111/cas.13864

28. Cheng Z, Ito S, Nishio N, et al.: Establishment of induced pluripotent stem cells from aged mice using bone marrow-derived myeloid cells. J Mol Cell Biol. 2011, 3:91-98. 10.1093/jmcb/mjq044 